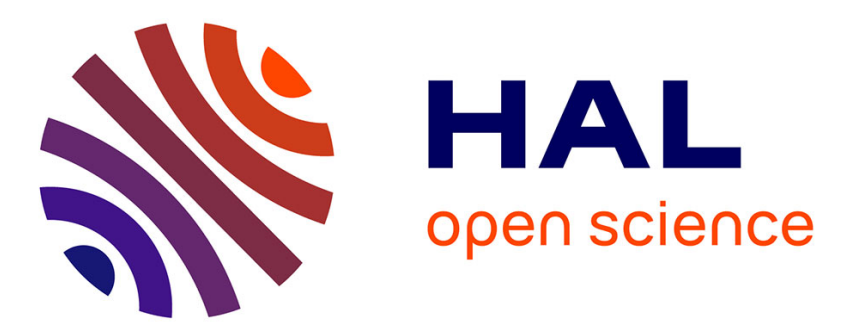

\title{
A Survey of Positioning Techniques and Location Based Services in Wireless Networks
}

Mohamad Yassin, Elias Rachid

\section{To cite this version:}

Mohamad Yassin, Elias Rachid. A Survey of Positioning Techniques and Location Based Services in Wireless Networks. IEEE 2015 Int. Conf. Signal Processing, Informatics, Communication and Energy Systems, Feb 2015, Kozhikode, India. 10.1109/SPICES.2015.7091420 . hal-01310662

\section{HAL Id: hal-01310662 \\ https://hal.inria.fr/hal-01310662}

Submitted on 3 May 2016

HAL is a multi-disciplinary open access archive for the deposit and dissemination of scientific research documents, whether they are published or not. The documents may come from teaching and research institutions in France or abroad, or from public or private research centers.
L'archive ouverte pluridisciplinaire HAL, est destinée au dépôt et à la diffusion de documents scientifiques de niveau recherche, publiés ou non, émanant des établissements d'enseignement et de recherche français ou étrangers, des laboratoires publics ou privés. 


\title{
A Survey of Positioning Techniques and Location Based Services in Wireless Networks
}

\author{
Mohamad Yassin* ${ }^{*}$, Elias Rachid* \\ *Saint-Joseph University, ESIB, Campus des Sciences et Technologies, Mar Roukoz, Lebanon \\ ‡University of Rennes 1, IRISA, Campus de Beaulieu, 35042 Rennes, France
}

\begin{abstract}
Positioning techniques are known in a wide variety of wireless radio access technologies. Traditionally, Global Positioning System (GPS) is the most popular outdoor positioning system. Localization also exists in mobile networks such as Global System for Mobile communications (GSM). Recently, Wireless Local Area Networks (WLAN) become widely deployed, and they are also used for localizing wireless-enabled clients. Many techniques are used to estimate client position in a wireless network. They are based on the characteristics of the received wireless signals: power, time or angle of arrival. In addition, hybrid positioning techniques make use of the collaboration between different wireless radio access technologies existing in the same geographical area. Client positioning allows the introduction of numerous services like real-time tracking, security alerts, informational services and entertainment applications. Such services are known as Location Based Services (LBS), and they are useful in both commerce and security sectors. In this paper, we explain the principles behind positioning techniques used in satellite networks, mobile networks and Wireless Local Area Networks. We also describe hybrid localization methods that exploit the coexistence of several radio access technologies in the same region, and we classify the location based services into several categories. When localization accuracy is improved, positiondependant services become more robust and efficient, and user satisfaction increases.
\end{abstract}

Index Terms-Positioning techniques; GPS; GSM; Wi-Fi; Location Based Services; hybrid positioning systems

\section{INTRODUCTION}

Numerous geolocation technologies are used to estimate client (person or object) geographical position. The large diversification of existing wireless Radio Access Technologies (RAT) and the increasing number of wirelessenabled devices are promoting the extensive application of Location Based Services (LBS) [1]. Position-dependant services include: emergency services such as rescue response [2] and security alerts, entertainment services like mobile gaming [3], medical applications [4] and a wide variety of other applications.

Global Positioning System (GPS) is the most common technology which supports outdoor locating services [5]. Satellites orbiting around the Earth continuously broadcast their own position and direction. Broadcasted signals are used by the receivers to estimate satellites positions as well as the distance between satellite and receiver. Having these distance measurements, trilateration [6] is usually used to estimate receivers position. Accuracy of the estimated positions depends on the number of visible satellites. Hence, it does not work well for indoor positioning, and it depends on weather conditions.

Base stations of the mobile terrestrial radio access networks, such as Global System for Mobile communi- cations (GSM), are the reference points for mobile client localization. Cell-Identification (Cell-ID) estimates client position using the geographical coordinates of its serving base station in cellular networks [7-9]. Other positioning methods are based on the fingerprinting database, and they make use of Received Signal Strength (RSS) measurements [10-13]. However, positioning accuracy is restrained by interference, multipath and non line-of-sight (NLOS) propagation.

A Wireless Local Area Network (WLAN) offers connectivity and internet access for wireless enabled clients within its coverage area. For example, IEEE 802.11 [14] (commonly known as Wi-Fi) is widely deployed, and it is also used for localizing Wi-Fi enabled devices. The fingerprinting approach is often used in Wi-Fi positioning systems $[15,16]$. It is based on Received Signal Strength Indication (RSSI) measurements in the localization area. Positioning systems using Wi-Fi are considered cost effective and practical solutions for indoor location tracking and estimation [17].

Positioning techniques performance comparison is done using several metrics [8] such as applicability, latency, reliability, accuracy and cost. A technique is more accurate when the estimated position of a client is closer to the real geographical position. Positioning accuracy is getting more important with the increasing use of position dependant applications. Indeed, it is crucial for emergency location services. Hence, hybrid positioning systems, such as $[18,19]$, are introduced to improve accuracy and reliability of the existing localization technologies.

In this paper, we describe the main positioning techniques used in satellite networks like GPS, in mobile networks, such as GSM, and in wireless local area networks such as Wi-Fi. The coexistence of several wireless radio access technologies in the same area allows the introduction of hybrid positioning systems, and promotes the diversification of position dependant services. We explain some of the hybrid localization techniques that coordinate information received from different radio access technologies in order to improve positioning accuracy. Such improvements increase user satisfaction, and make LBS more robust and efficient. We also classify these services into several categories.

The rest of the paper is organized as follows: in (II) we explain the principles behind positioning techniques used in satellite and mobile networks. Wi-Fi localization methods are reported in (III). We describe hybrid positioning systems in (IV). Section (V) contains a classification of LBS. Concluding remarks are given in section (VI). 


\section{Positioning in GPS And Mobile Networks}

GPS consists of a network of 24 satellites in six different 12-hour orbital paths spaced so that at least five are in view from every point on the globe [20]. Satellites serve as reference points when estimating client position. They continuously broadcast signals containing information about their own position and direction. Distance between satellite and receiver is determined by precisely measuring the time it takes a signal to travel from the satellite to the receivers antenna.

Once the distances between visible satellites and the GPS receiver are measured, client position is estimated via the trilateration method, commonly known as triangulation [5]. Three distance measurements are required to perform position estimation. In fact, the estimated position is the intersection of three spheres having the satellites as centers and the calculated distances as radii.

GPS accuracy is largely reduced by several factors such as signal delays, satellite clock errors, multipath distortion, receiver noise and various environment noises [21]. To overcome visibility problems between satellites and receivers, assisted-GPS is proposed. It benefits from the coexistence of satellite networks along with terrestrial wireless access networks (i.e. mobile networks or Wireless Local Area Networks) in the same area. Therefore, superior accuracy, availability and coverage are offered for indoor use or in urban areas. Refer to [22] for more information about Assisted-Global Positioning System.

In mobile networks, such as GSM, many techniques are used to estimate client position. Contrarily to the satellites that are continuously moving around the globe, base stations (BS) of the mobile networks have fixed geographical positions. In addition, each BS broadcasts its Cell-ID and Location Area Identifier (LAI) to the mobiles within its coverage area. Therefore, each mobile can approximate its own position using the geographical coordinates of its serving base station in Cell-ID method [9].

Angle of Arrival (AoA) measurements [23, 24] of several radio links between the base stations and the mobile are also used to estimate client position. Hence, user position is approximated according to these angle measurements and using information about base stations geographical coordinates. Time of Arrival (ToA) [25, 26] requires synchronization between the different network elements (i.e., base stations and mobile stations). The time difference between bursts sent by the mobile are converted into distance. Hence, trilateration is used to estimate client position. Other methods use received signal strength measurements to localize mobile stations. For example, received signal power is converted into distance via propagation models or empirical models. In addition, the fingerprinting method [27] compares RSS measurements with the values stored in a database for specific points in the localization map in order to approximate client position.

Time Difference of Arrival (TDoA) [28, 29] technique is inspired by ToA. Indeed, in ToA, the positioning entity measures signal propagation time from the emitter to the receiver. Time measurement is converted into distance that is used to estimate client position. However, TDoA technique requires the simultaneous transmission (for each base station) of two signals having different frequencies. These signals will reach the receiver at different times. Therefore, the time difference is measured and converted into distance. Once we have three distance measurements, trilateration is used to estimate client position. Enhanced Observed Time Difference (E-OTD) [30] requires synchronization between network entities (base stations and mobiles). Each base station broadcasts messages within its coverage area. A mobile station compares the relative times of arrival of these messages to estimate its distance from each visible base station.

Localization techniques in mobile networks can be classified in two categories: network-based and clientbased. In network-based positioning techniques, the network collects necessary information to estimate client position. Time, angle or distance measurements performed by the base stations are usually forwarded to a positioning server deployed in the network. The required information when estimating user position is stored in the positioning database. Thus, positioning server has information about the positions of all the users in the system. However, client-based localization techniques are characterized by the absence of a centralized positioning entity. In fact, each client performs time, angle, power or distance measurements locally. Thus, it approximates its own position using local measurements and information broadcasted from the base stations.

Fig. 1 shows a qualitative comparison of the positioning techniques described in this section. Performance criteria used for localization techniques comparison are accuracy and coverage. A positioning technique is better when it has lower accuracy error (distance between the estimated position and the real geographical position) and greater coverage.

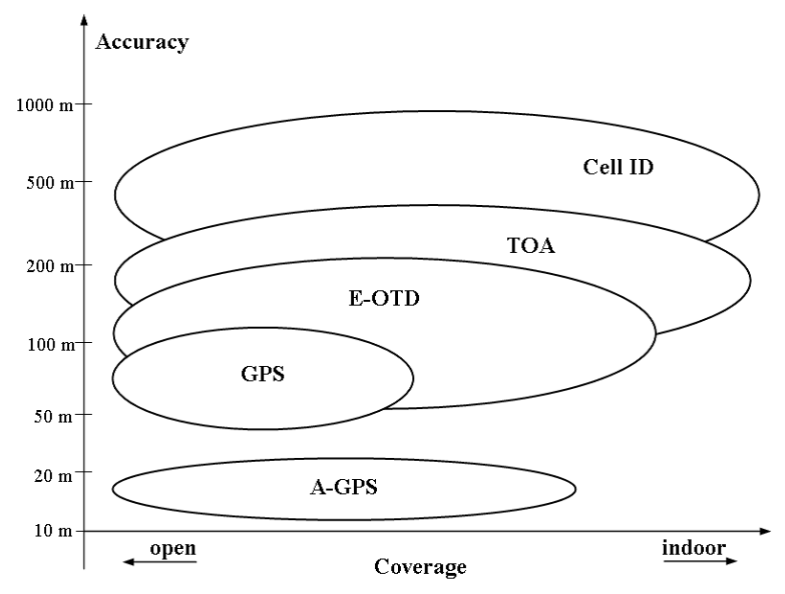

Fig. 1. Positioning techniques comparison [9]

\section{WLAN POSITIONING TECHNIQUES}

The increasing number of Internet users, the recent development of wireless technologies and the proliferation of mobile and web applications have boosted the implementation of WLANs. For instance, the IEEE 802.11 standard [14] (commonly known as Wi-Fi) is widely 
deployed nowadays. The base station of a Wi-Fi network is called "Access Point." Fig. 2 shows three access points serving several Wi-Fi clients located within their coverage area.

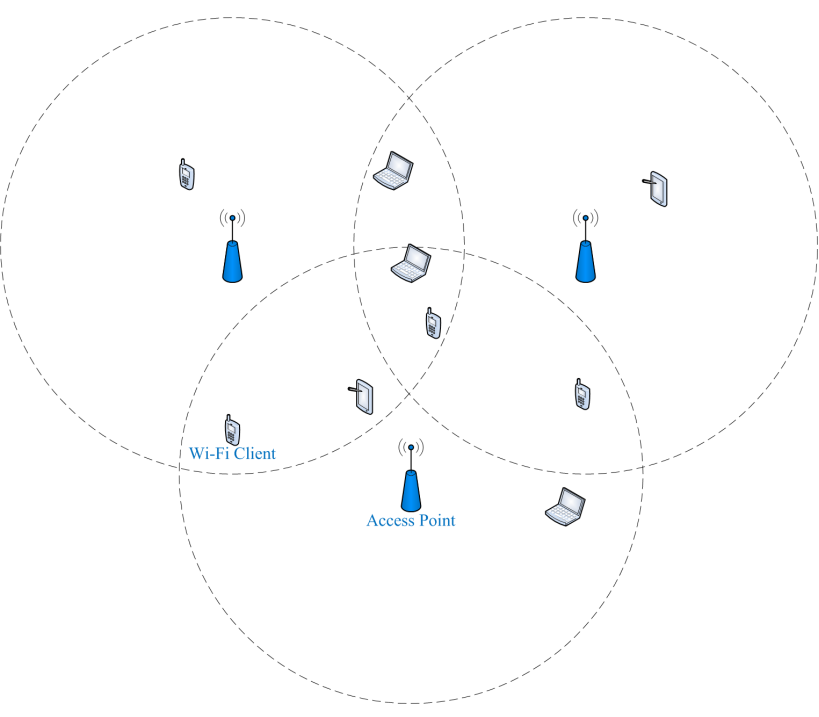

Fig. 2. Wi-Fi network having several clients

Traditionally, a Wi-Fi network provides Internet access to wireless-enabled clients located within its coverage area. In addition, it allows interconnectivity between wireless devices existing in the same network. Recently, Wi-Fi networks are having additional applications. For example, we can benefit of the coexistence of several radio access technologies in the same geographical area. Heterogeneous networks offer the possibility to steer user sessions preferentially to a given Radio Access Technology, such as Wi-Fi or Universal Mobile Terrestrial radio access System (UMTS), according to service type [31] and network load. Moreover, the wide deployment of Wi-Fi networks allows the introduction of numerous location-based services.

Wi-Fi positioning techniques are similar to those used in mobile networks. However, the most common technique used to localize a client in Wi-Fi networks is based on RSSI measurements. In the remainder of this section, we classify Wi-Fi positioning techniques into several categories, and we describe the basics of RSSI-based localization methods.

ToA [32] and TDoA perform time measurements to calculate the distance between $\mathrm{Wi}-\mathrm{Fi}$ client and access points. Hence, three distance measurements are required to estimate user position via trilateration [33]. Such methods belong to the category of time-based positioning techniques, and they require time synchronization between network entities. In Cell-ID category, users scan the received radio beacons to estimate the closer access point. They use either predefined radio propagation models or experimental fingerprinting data to estimate user position.

AoA method uses directional antennas to measure the angle of arrival of signals transmitted by the clients. Hence, client position is estimated via the geometry of triangles in angle-based positioning techniques. However, the most common positioning techniques in Wi-Fi networks are based on RSSI measurements [34]. Some of them are based on propagation models [35] to translate signal power into distance. Other methods use empirical models, and they store RSSI measurements in a positioning database. Therefore, localization methods in $\mathrm{Wi}-\mathrm{Fi}$ are classified into four main categories: Cell-ID, time, RSSI and angle. Fig. 3 illustrates the classification of Wi-Fi positioning techniques.

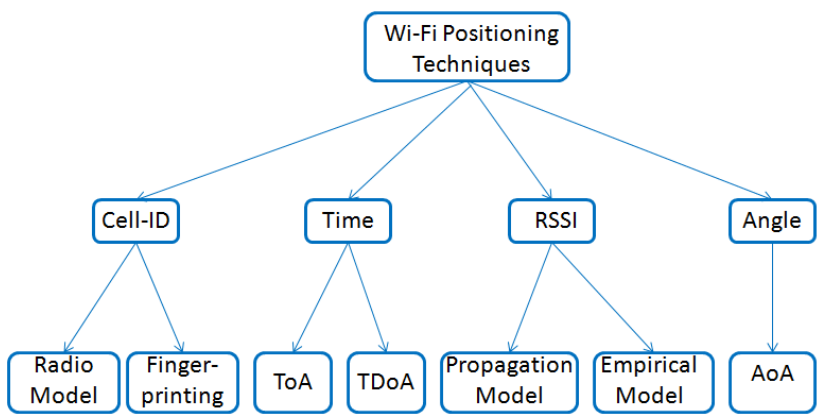

Fig. 3. Categories of $\mathrm{Wi}-\mathrm{Fi}$ positioning techniques

Received signal strength indication measurements are quantified levels that reflect the real power of a received wireless signal. When propagating in free space, the transmitted radio frequency signal is subject to degradation due to attenuation, reflection, diffraction and scattering. Indeed, several propagation models formulate signal strength degradation as a function of the traveled distance and the transmission frequency. For instance, Hata-Okumura [36] model approximates Path Loss (PL) according to the distance between emitter and receiver, antenna characteristics and transmission frequency. Hence, RSSI measurements are compared with theoretical values of the received power (calculated using propagation models) in order to find the distance traveled by the signal. Three distance measurements are required to estimate Wi-Fi-enabled client position via trilateration. In fact, it is the intersection of three circles having the access points as centers and the calculated distances as radii.

Other positioning techniques based on RSSI measurements use empirical models to estimate user position. Instead of approximating the distance between Wi-Fi clients and access points, the localization area is divided into smaller parts using a grid. Each point of the grid receives several Wi-Fi signals from the neighboring access points. RSSI measurements are performed under different conditions (e.g. time, interference, network load) in order to increase positioning accuracy. If $n$ is the number of WiFi access points, an $n$-tuple $\left(\overline{R S S I_{1}}, \overline{R S S I_{2}}, \ldots, \overline{R S S I_{n}}\right)$ containing mean RSSI values is created for each point $(x, y)$ in the map. Such positioning technique is called RSSI fingerprinting, and it occurs in two phases: an offline fingerprinting phase and an online positioning phase.

In the first phase, RSSI measurements are done for each point in the positioning map (under different network conditions). At the end of this phase, positioning database is created. It contains mean RSSI values for every point in the grid [37]. However, the second phase performs online RSSI measurements for signals received from the neighboring access points. The positioning entity compares live RSSI measurements with the values stored in the database. Therefore, client position is estimated as the entry $(x, y)$ 
in the database that best matches the actual measurements [38]. Accuracy of RSSI-based positioning techniques in Wi-Fi networks depends on the number of access points involved in the localization problem.

\section{Hybrid Positioning System}

The wide usage of position dependant services increases the need for more accurate position estimation techniques. Due to the limitations of positioning methods that use data from one single RAT, hybrid positioning techniques are proposed to increase accuracy. They make use of the collaboration between different wireless access networks existing in the same geographical area, such as GPS and GSM, to exchange additional position related data.

The main factors that reduce accuracy of GPS are multipath distortion and visibility problem between satellites and receivers [20]. Therefore, a hybrid positioning technique called Assisted-Global Positioning System (A-GPS) [22] is introduced to overcome these limitations. In fact, GSM Base Transceiver Stations (BTS) are involved in the positioning problem along with the satellites. Additional data about BTS geographical position and proximity to the mobile is used together with GPS localization information in order to estimate client position. Moreover, authors of [39] propose a Wi-Fi GPS based combined positioning algorithm. Indeed, localization data from the Wi-Fi network is used when the number of visible satellites is less than four.

In cellular networks, positioning accuracy is restrained by the non-line-of-sight propagation and by interference mitigation techniques. Thus, inconsistent information is provided as entry when resolving the localization problem. Authors of [18] describe a hybrid positioning scheme that combines TDoA technique measurements obtained from the cellular network with GPS range information to improve the accuracy of mobile client position estimation. The obtained accuracy should be within the limits required by emergency location services.

ToA technique is very frequently used for positioning in mobile networks. However, it requires synchronization between mobiles and base stations. In addition, ToA is restricted by multipath and non-line-of-sight propagation problems. Hence, this positioning technique is assisted by additional information from AoA technique. Angle measurements are performed by the serving base station using antenna arrays. The usage of ToA assisted AoA technique improves positioning accuracy, especially in bad propagation environments [26].

In wireless local area networks, such as Wi-Fi, RSSI fingerprinting technique depends on the number of access points involved in the localization process. The validity of mean RSSI measurements stored in the database also affects positioning accuracy. We exploit the coexistence of Personal Area Networks (PAN) along with Wi-Fi in the same geographical area to improve positioning accuracy. In fact, RSSI measurements are done for all the existing wireless technologies, and results are stored in the positioning database. Therefore, Wi-Fi fingerprinting uses additional RSSI information from wireless PANs existing in the same area such as Bluetooth and ZigBee networks.
More information about this hybrid technique is found in [38].

\section{LOCATION BASED SERVICES}

In wireless networks, the knowledge of user geographical position allows the introduction of numerous position dependant applications. These applications are known as location-based services, and they are useful for service providers as well as for mobile clients.

The wide deployment of radio access technologies and the increasing development of wireless-enabled devices are promoting the extensive use of LBS. As mentioned in the previous sections, numerous positioning techniques are used to estimate client position. However, the main parameter for LBS efficiency is accuracy of the positioning technique. Indeed, users are more satisfied when the estimated position is closer to the real geographical position, and when the probability of erroneous estimations is reduced.

Location-based services are related to the position of the user making the request. They are classified [40] as emergency services (e.g. security alerts, public safety and query of the nearest hospital), informational services (i.e., news, sports, stocks and query of the nearest hotel or cinema), tracking services (like asset/fleet/logistic monitoring or person tracking), entertainment services (for example: locating a friend and gaming) and advertising services (such as announcements or invitation messages broadcasted by the shops to the nearby mobile clients). Moreover, future applications of LBS include support to the studies on climate change, seismology and oceanography.

Position dependant services are useful for mobile mapping, deformation monitoring and many civil engineering applications [41]. They have revolutionized navigation (on land, in the air and at sea) and intelligent transportation system by increasing their safety and efficiency. However, user location privacy security poses a potentially grave threat [1]. In fact, it is possible to access user location information anytime and anywhere. Therefore, many privacy protection methods are introduced to deal with the contradiction between location privacy protection and quality of service in LBS. Some of them protect user ID information by hiding the true ID when requesting the service. Other methods do not submit the exact location to the server, but they send a region containing user exact position.

\section{CONCLUSION}

In this paper, we surveyed the major positioning techniques used in wireless networks. Global positioning system is the most common technology for outdoor locating services. Localization techniques in mobile networks, such as global system for mobile communications, perform time, angle or signal power measurements to estimate user position. In Wi-Fi networks, received signal strength indication fingerprinting approximates user position within the coverage area. Accuracy depends on the number of access points involved in the positioning process.

Hybrid positioning techniques are proposed to satisfy the increasing need for positioning accuracy. They make 
use of the collaboration between multiple radio access technologies existing in the same geographical area. For instance, Assisted-GPS uses information from the mobile network to improve positioning accuracy in GPS. Location-based services are based on the knowledge of user position. They include emergency, informational, tracking, entertainment and advertising services. LBS are useful for service providers and for the clients. Accuracy improvements increase user satisfaction, and make the localization services more robust and efficient.

\section{REFERENCES}

[1] W. Zhang, X. Cui, D. Li, D. Yuan, and M. Wang, "The Location Privacy Protection Research in Location-Based Service," in $18^{\text {th }}$ Int. Conf. Geoinformatics, Beijing, 2010.

[2] J. Casper and R. R. Murphy, "Human-Robot Interactions During the Robot Assisted Urban Search and Rescue Response at the World Trade Center," in IEEE Trans. Systems, Man and Cybernetics, Part $B$, pp. 367-385, June 2003

[3] P. Ferreira, J. Orvalho, and F. Boavida, "A Middleware Architecture for Mobile and Prevasive Large Scale Augmented Reality Games," in Proc. Conf. Networks and Services Research, 2007.

[4] S. Hong, M. H. Lee, H. H. Chun, S. H. Kwon, and J. L. Speyer, "Observability of Error States in GPS/INS Integration," in IEEE Trans. Vehicular Technology, 2005.

[5] S. C. Yeh, W. H. Hsu, M. Y. Su, C. H. Chen, and K. H. Liu, "A Study on Outdoor Positioning Technology Using GPS and WiFi Networks," in Int. Conf. Networking, Sensing and Control, Okayama, 2009.

[6] B. T. Fang, "Trilateration and Extension to Global Positioning System Navigation," in J. Guidance, Control and Dynamics, pp. 715717, 1986.

[7] T. Kos, M. Grgic, and G. Sisul, "Mobile User Positioning in GSM/UMTS Cellular Networks," in $48^{\text {th }}$ Int. Symp. Multimedia Signal Processing and Communications, Zadar, 2006.

[8] I. K. Adusei, K. Kyamakya, and K. Jobmann, "Mobile Positioning Technologies in CellularNetworks: an Evaluation of their Performance Metrics," in Proc. Military Communications Conf., 2002.

[9] E. Trevisani and A. Vitaletti, "Cell-ID Location Technique Limits and Benefits: an Experimental Study," in Proc. $6^{\text {th }}$ IEEE Workshop Mobile Computing Systems and Applications, 2004.

[10] H. Laitinen, J. Lahteenmaki, and T. Nordstorm, "Database Correlation Method for GSM Location," in Proc. IEEE Vehicular Technology Conf., 2001.

[11] J. Talvitie and E. S. Lohan, "Modeling Received Signal Strength Measurements for Cellular Network Based Positioning," in Int. Conf. Localization and GNSS, Turin, 2013.

[12] N. Deblauwe and L. V. Biesen, "Cellular Positioning: from Received Signal Strength Measurements to the Algorithm," in IEEE Global Communications Conf., New Orleans, 2008.

[13] C. M. Huang, T. H. Hsieh, and S. Y. Lin, "A SignalAware Fingerprinting-Based Positioning Technique in Cellular Networks," in $14^{\text {th }}$ Int. Conf. Network-Based Information Systems, Tirana, 2011.

[14] S. G. Matthew, 802.11 Wireless Networks: The Definitive Guide, OReilly, 2002

[15] T. Vaupel, J. Seitz, F. Kiefer, S. Haimerl, and J. Thielecke, "Wi-Fi Positioning: System Considerations and Device Calibration," in Int. Conf. Indoor Positioning and Indoor Navigation, Zurich, 2010.

[16] B. Shin, J. H. Lee, T. Lee, and H. S. Kim, "Enhanced Weighted K-Nearest Neighbor Algorithm for Indoor Wi-Fi Positioning Systems," in $8^{\text {th }}$ Int. Conf. Computing Technology and Information Management, Seoul, 2012.

[17] N. Chang, R. Rashidzadeh, and M. Ahmadi, "Robust Indoor Positioning Using Differential Wi-Fi Access Points," in IEEE Trans. Consumer Electronics, 2010.

[18] G. De Angelis, G. Baruffa, and S. Cacopardi, "GNSS/Cellular Hybrid Positioning System for Mobile Users in Urban Scenarios," in IEEE Trans. Intelligent Transportation Systems, vol. 14, no. 1, Mar. 2013.

[19] S. Rooney, P. Chippendale, R. Choony, C. Le Roux, and B. Honary, "Accurate Vehicular Positioning Using a DAB-GSM Hybrid System," in IEEE 51 ${ }^{\text {st }}$ Vehicular Technology Conf. Proc., Tokyo, 2000.

[20] R. Bajaj, S. L. Ranaweera, and D. P. Agrawal, "GPS: LocationTracking Technology," in Computer, vol. 35, no. 4, pp. 92-94, Apr. 2002.
[21] J. Y. Huang and C. H. Tsai, "Improve GPS Positioning Accuracy with Context Awareness," in The $1^{\text {st }}$ IEEE Int. Conf. Ubi-Media Computing, Lanzhou, 2008.

[22] G. M. Djuknic and R. E. Richton, "Geolocation and Assisted GPS," in Computer, vol. 34, no. 2, pp. 123-125, Aug. 2002.

[23] A. Pages-Zamora, J. Vidal, and D. H. Brooks, "Closed-Form Solution for Positioning Based on Angle of Arrival Measurements," in The $13^{\text {th }}$ IEEE Int. Symp. Personal, Indoor and Mobile Radio Communications, 2002.

[24] D. Niculescu and B. Nath, "Ad-Hoc Positioning System (APS) Using AoA," in Proc. $22^{\text {nd }}$ Annu. Joint Conf. IEEE Computer and Communications, 2003.

[25] M. T. Simsim, N. M. Khan, R. Ramer, and P. B. Rapajic, "Time of Arrival Statistics in Cellular Environments," in Proc. IEEE $63^{\text {rd }}$ Vehicular Technology Conf., 2006.

[26] P. Deng and P. Z. Fan, "An AoA Assisted ToA Positioning System," in Int. Conf. Communication Technology Proc., Beijing, 2000.

[27] H. Laitinen, J. Lahteenmaki, and T. Nordstorm, "Database Correlation Method for GSM Location," in Proc. IEEE Vehicular Technology Conf., 2001.

[28] D. H. Shin and T. K. Sung, "Comparisons of Error Characteristics Between ToA and TDoA Positioning," in IEEE Trans. Aerospace and Electronic Systems, 2002.

[29] F. Gustafsson and F. Gunnarsson, "Positioning Using TimeDifference of Arrival Measurements," in Proc. IEEE Int. Conf. Acoustics, Speech and Signal Processing, 2003.

[30] S. Charitanetra and S. Noppanakeepong, "Mobile Positioning Location Using E-OTD Method for GSM Network," in Proc. Student Conf. Research and Development, 2003, pp. 319-324.

[31] J. Venes and L. M. Correia, "Performance of a Heterogeneous Network with UMTS, Wi-Fi and WiMAX," in IEEE Int. Conf. Computer as a Tool, Lisbon, 2011.

[32] M. Ciurana, S. Cugno, and F. Barcelo-Arroyo, "WLAN Indoor Positioning Based on ToA with Two Reference Points," in $4^{t h}$ Workshop Positioning, Navigation and Communication, Hannover, 2007.

[33] L. Peneda, A. Azenha, and A. Carvalho, "Trilateration for Indoors Positioning Within the Framework of Wireless Communications," in The $35^{\text {th }}$ Annu. Conf. IEEE Industrial Electronics, Porto, 2009.

[34] M. Yassin, E. Rachid, and R. Nasrallah, "Performance Comparison of Positioning Techniques in Wi-Fi Networks," in $10^{\text {th }}$ Int. Conf. Innovations in Information Technology, Al Ain, 2014.

[35] P. Chai and L. Zhang, "Indoor Radio Propagation Models and Wireless Network Planning," in IEEE Int. Conf. Computer Science and Automation Engineering, Zhangiajie, 2012.

[36] M. Kumar, V. Kumar, and S. Malik, "Performance and Analysis of Propagation Models for Predicting RSS for Efficient Handoff," in Int. J. Advanced Scientific and Technical Research, 2012.

[37] M. Hassan-Ali and K. Pahlavan, "A New Statistical Model for SiteSpecific Indoor Radio Propagation Prediction Based on Geometric Optics and Geometric Probability," in IEEE Trans. Wireless Commun., 2002.

[38] M. L. Rodrigues, L. F. Vieira, and M. Campos, "FingerprintingBased Radio Localization in Indoor Environments Using Multiple Wireless Technologies," in IEEE $22^{\text {nd }}$ Symp. Personal, Indoor and Mobile Radio Communications, Toronto, 2011.

[39] S. Zirari, P. Canalda, and F. Spies, "Wi-Fi GPS Based Combined Positioning Algorithm," in IEEE Int. Conf. Wireless Communications, Networking and Information Security, Beijing, 2010.

[40] D. Mohapatra and S. B. Suma, "Survey of Location Based Wireless Services," in IEEE Int. Conf. Personal Wireless Communications, 2005.

[41] J. -j. Peng, "A Survey of Location Based Service for Galileo System," in Int. Symp. Computer Science and Computational Technology, Shanghai, 2008. 